\title{
Level-Rank Duality of WZW Models in Conformal Field Theory
}

\author{
Tomoki Nakanishi and Akihiro Tsuchiya \\ Department of Mathematics, Nagoya University, Chikusa-ku, Nagoya, 464, Japan
}

Received August 13, 1990

\section{Dedicated to Professor Masahisa Adachi on his 60th birthday}

\begin{abstract}
We consider the decomposition of the conformal blocks under the conformal embeddings. The case $\widehat{g l}(l r)_{1} \supset \widehat{s l}(l)_{r} \times \widehat{s l}(r)_{l} \times \hat{a}(\hat{a}$ is an affine extension of the abelian subalgebra of the central elements of $g l(l r))$ is studied in detail. The reciprocal decompositions of $\widehat{g l}(l r)_{1}$-modules induce a pairing between the spaces of conformal blocks of $\widehat{s l}(l)_{r}$ and $\widehat{s l}(r)_{l}$ Wess-Zumino-Witten models on the Riemann sphere. The completeness of the pairing is shown. Hence it defines a duality between two spaces.
\end{abstract}

\section{Introduction}

The curiosity about the affine Lie algebras pair $\widehat{s l}(l)_{r}$ and $\widehat{s l}(r)_{l}$ have appeared in several contexts. In [F], it was found that there is a mutually commutative embedding of affine Lie algebras

$$
\widehat{s l}(l)_{r} \times \widehat{s l}(r)_{l} \subset \widehat{g l}(l r)_{1} .
$$

Remarkably, mutually transposed pairs of representations $Y \otimes{ }^{t} Y$ of $\widehat{s l}(l)_{r} \times \widehat{s l}(r)_{l}$ appeared in the reciprocal decomposition of irreducible representations of $\widehat{g l}(l r)_{1}[\mathrm{~F}]$, and the decomposition formula for the character of $\widehat{g l}(l r)_{1}[\mathrm{JM}]$. The branching rule of the embedding (1.1) was studied in $[\mathrm{H}, \mathrm{ABI}]$ in detail.

For any reductive affine Lie algebra, one can associate a Wess-Zumino-Witten (WZW) model [Wi] using the Sugawara construction [KZ, GWi]. Recently, two direct connections between $\widehat{s l}(l)_{r}$ and $\widehat{s l}(r)_{l}$ WZW models were found: The first one is an equality between the fusion rules of the both models proved in [KN, GWe] and partially proved in [SA]:

$$
N_{Y_{1} Y_{2}}^{Y_{3}} \widehat{s l}(l)_{r}=N_{t_{Y_{1}}{ }^{t} Y_{2}}^{t} \widehat{s l}(l)_{l}
$$

where the Young diagrams $Y_{i}$ representing the primary fields, their sizes $\left|Y_{i}\right|$ are related as $\left|Y_{1}\right|+\left|Y_{2}\right|=\left|Y_{3}\right|$ and ${ }^{t} Y$ is the transposition of $Y$. The second one is strong evidence of the existence of a duality relation between the conformal blocks of 4-point functions of both models [NS]. 
In this article, we show a duality relation between the spaces of conformal blocks of $\widehat{s l}(l)_{r}$ and $\widehat{s l}(r)_{l}$ WZW models on the Riemann sphere using the theory of conformal blocks developed in [TK, TUY, FS, MS]. To construct the duality, we use a conformal embedding [GR, G, GNO]

$$
\widehat{s l}(l)_{r} \times \widehat{s l}(r)_{l} \times \hat{a} \subset \widehat{g l}(l r)_{1},
$$

where $\hat{a}$ is an affine extension of the abelian subalgebra of the central elements of $g l(l r)$. The embedding (1.3) induces the decomposition of the $\widehat{g l}(l r)_{1}$ conformal blocks into the sum of the $\widehat{s l}(l)_{r} \times \widehat{s l}(r)_{l} \times \hat{a}$ conformal blocks:

$$
\varphi: \mathscr{V}_{\vec{p}}^{G \dagger} \rightarrow \bigoplus_{\vec{\lambda}, \vec{\lambda}^{\prime}} \mathscr{V}_{\vec{\lambda}}^{L \dagger} \otimes \mathscr{V}_{\vec{\lambda}^{\prime}}^{R \dagger}, \otimes \mathscr{V}_{\vec{p}}^{A \dagger},
$$

where $\mathscr{T}_{\vec{\mu}}^{X \dagger}(X=G, L, R, A)$ denotes the space of the conformal blocks of $\widehat{g l}(l r)_{1}$, $\widehat{s l}(l)_{r}, \widehat{s l}(r)_{l}, \hat{a}$ having representations $\vec{\mu}=\left(\mu_{1}, \ldots, \mu_{N}\right)$ as the external states (see Sect. 2 for the definition). Because the dimensions of the conformal blocks of $\widehat{g l}(l r)_{1}$ and $\hat{a}$ WZW models on the Riemann sphere are at most one, the domposition (1.4) determined the coefficient matrix $C\left(\vec{p}, \vec{\lambda}, \vec{\lambda}^{\prime}\right)_{\alpha \beta}$ by taking bases of $\mathscr{T}_{\vec{\lambda}}^{L \dagger}$ and $\mathscr{V}_{\vec{\lambda}^{\prime}}^{R \dagger}$. The equivalence of the energy-momentum tensors of both sides of (1.3) guarantees that this matrix is independent of the insertion points of the external states.

Our main assertion in this paper (Sect. 4, Theorem) is a fact

$$
\operatorname{det} C\left(\vec{p}, \vec{\lambda}, \vec{\lambda}^{\prime}\right) \neq 0
$$

if $\vec{\lambda}$ and $\vec{\lambda}^{\prime}$ is included in the branching rule of $\vec{p}$, and the dimensions of the space of the conformal blocks $\mathscr{V}_{\vec{\lambda}}^{L \dagger}, \mathscr{V}_{\vec{\lambda}^{\prime}}^{R \dagger}$ are not zero. The non-singular matrix (1.5) then determines a duality between two spaces $\mathscr{V}_{\vec{\lambda}}^{L \dagger}$ and $\mathscr{V}_{\vec{\lambda}^{\prime}}^{R \dagger}$, which justify the observation of [NS]. If all of the external fields of the conformal blocks of $\widehat{g l}(l r)_{1}$ are the same, it is invariant under the braid group. It follows that the braid matrices of both invariant subspaces of $\widehat{s l}(l)_{r}$ and $\widehat{s l}(r)_{l}$ conformal blocks are contragradient to each other up to a phase factor (Sect. 4, Proposition). A key fact for our proof of (1.5) is the irreducibility of the conformal blocks of $\widehat{s l}$ under the braid group if all the external fields are those of the vector representation. To prove the general case, we shall extensively use the commutativity of the operations of factorization and the decomposition, which is a consequence of the equivalence of the energy-momentum tensors of the conformal embedding (1.3).

In Sect. 2, we formulate the decomposition of the conformal blocks of the $\hat{g}$ WZW model into those of $\hat{h}$ WZW model for a conformal embedding $\hat{g} \supset \hat{h}$. In Sect. 3 , we prepare several facts about the conformal embedding (1.3) realized on the free fermion Fock space. Especially, the highest weight states of $\widehat{s l}(l)_{r} \times \widehat{s l}(r)_{l} \times \hat{a}$ and the branching rule are reviewed. In Sect.4, we derive a pairing between the spaces of conformal blocks of $\widehat{s l}(l)_{r}$ and $\widehat{s l}(r)_{l}$ WZW models, and give its consequences on the braid matrices of these dual conformal blocks. In Sect. 5, we give a proof of the completeness of the pairing. In Sect. 6 we discuss further extensions of the duality. The conformal blocks of $\widehat{g l}(l r)_{1}$ and $\hat{a}$ are given in the appendix.

We remark recent relevant works. The level-rank duality appears in the quantum groups $U_{q}(s l(l))$ and $U_{q}(s l(r))$ at $l+r^{\text {th }}$ root of unity [FD, SA, NRS]. The duality is 
also related to non-diagonal modular invariants of WZW models [Wa, ABI]. There is a closely related work [GWe] in which the level-rank duality was studied in the Hecke algebra. There are other branches of physical systems where the level-rank duality plays important roles such as the two dimensional integrable lattice models called the restricted solid-on-solid (RSOS) models [JMO, KN, KNS, BR], the ChernSimons topological field theory [NRS], a series of non-unitary minimal models of $W_{n}$ algebra and their deformations [KNS, ABS].

\section{Decomposition of the Conformal Blocks for Conformal Embeddings}

An affine Lie algebra pair $\hat{g} \supset \hat{h}$ is called a conformal embedding if the central charges of their Virasoro subalgebras satisfy the equality

$$
c(\hat{g})=c(\hat{h}) .
$$

Such examples are initiated by [GO, GNO] and studied in the context of the string model building [BETZ]. The complete classification was given in [BB, SW]. The important feature of the conformal embedding is that the energy-momentum tensor of both models coincide, i.e.,

$$
T_{\hat{g}}(z)=T_{\hat{h}}(z) .
$$

Below we consider the decomposition of the conformal blocks of the $N$-point functions on the Riemann sphere of the $\hat{g}$ WZW model by the one of the $\hat{h}$ WZW model for a conformal embedding $\hat{g} \supset \hat{h}$. As for the formulation of conformal blocks, we follow the one in [TK, TUY] and consult them in further detail.

Let $Z=\left(z_{i}\right)_{i=1}^{N}$ be the collection of the $N$ different points on the sphere and attach an integrable representation $\lambda_{i}$ for each point $z_{i}$. $L_{\lambda}$ denotes the representation space of $\lambda$. Let us introduce the dual space $L_{\vec{\lambda}}^{\dagger}=\left(\bigotimes_{i=1}^{N} L_{\lambda_{2}}\right)^{*}$. From the set of currents $J_{I}(z)$ associated to an orthogonal basis of a simple Lie algebra $g$, the energy momentum tensor of $\hat{g}$ is defined through the Sugawara construction [KZ]

$$
\begin{aligned}
T(z) & =\frac{1}{2\left(g^{*}+l\right)} \sum_{I=1}^{\mathrm{rank}}: J_{I}(z) J_{I}(z): \\
& =\lim _{w \rightarrow z} \frac{1}{2\left(g^{*}+l\right)} \sum_{I}\left\{J_{I}(w) J_{I}(z)-(z-w)^{-2}\right\}
\end{aligned}
$$

with $g^{*}$ and $l$ the dual Coxeter number and the level of $\hat{g}$ respectively. For a general reductive $\hat{g}$, the energy-momentum tensor is given by the sum of the energy-momentum tensors of its simple or abelian components.

A conformal block $\left\langle\Phi_{\vec{\lambda}}(Z)\right|$ is defined as follows [TUY]:

1) For each $Z,\left\langle\Phi_{\vec{\lambda}}(Z)\right|$ belongs to the dual space $L_{\vec{\lambda}}^{\dagger}$.

2) For each $Z,\left\langle\Phi_{\vec{\lambda}}(Z)\right|$ satisfies the gauge invariance condition. Namely, for any meromorphic function $\xi(w)$ on the sphere which is holomorphic outside the points $Z$, the following residue formula for the currents $J_{I}$ holds:

$$
\left\langle\Phi_{\vec{\lambda}}(Z)\right| \sum_{i=1}^{N} \operatorname{Res}_{z_{i}}\left[\xi(w) \varrho_{i}\left(J_{I}(w)\right) d w\right]=0
$$


Here $\varrho_{i}$ restricts the action of currents $J_{I}(w)$ only on the $i^{\text {th }}$ representation $\lambda_{i}$. 3) It satisfies the following differential equation

$$
\partial_{z_{i}}\left\langle\Phi_{\vec{\lambda}}(Z)\right|=\left\langle\Phi_{\vec{\lambda}}(Z)\right| \operatorname{Res}_{z_{i}} \varrho_{i}(T(w) d w) .
$$

We define the space $\mathscr{V}_{\vec{\lambda}}^{\dagger}(Z)$ as the subspace of $L_{\vec{\lambda}}^{\dagger}$ consisting of the elements satisfying the above condition 2) at $Z$. Also $\mathscr{T}_{\vec{\lambda}}^{\dagger}$ denotes the space of the conformal blocks.

Each $\hat{g}$ integrable representation $\hat{\lambda}_{i}$ decomposes into a finite number of irreducible components as an $\hat{h}$ representation like

$$
L_{\lambda_{i}} \cong \bigoplus_{\mu_{\imath}}\left(B\left(\lambda_{i} ; \mu_{i}\right) \otimes L_{\mu_{i}}\right)
$$

where the space of branching coeffients $B(\lambda ; \mu)$ is finite dimensional. Define the set $b(\vec{\lambda})=\left\{\vec{\mu}=\left(\mu_{1}, \ldots, \mu_{N}\right) \mid \operatorname{dim} B\left(\lambda_{i} ; \mu_{i}\right)>0\right.$ for all $\left.i\right\}$ and the space $B(\vec{\lambda} ; \vec{\mu})=\bigotimes_{i=1}^{N} B\left(\lambda_{i} ; \mu_{i}\right)$. Then there is an isomorphism

$$
\varphi: L_{\vec{\lambda}}^{\dagger} \rightarrow \bigoplus_{\vec{\mu} \in b_{\lambda}}\left(B^{*}(\vec{\lambda} ; \vec{\mu}) \otimes L_{\vec{\mu}}^{\dagger}\right)
$$

The main claim in this section is the following decomposition property of conformal blocks of $\hat{g}$ WZW model into those of $\hat{h}$ WZW model:

Let us choose bases $\left\{\left\langle\left.\Psi_{\vec{\lambda}}^{G}(Z)\right|_{\alpha}\right\}_{\alpha},\left\{\left\langle\left.\Psi_{\vec{\mu}}^{H}(Z)\right|_{\beta}\right\}_{\beta}\right.\right.$ of the spaces of the conformal blocks of $\hat{g}$ and $\hat{h}$ WZW models respectively, and also choose a basis $\left\{f(\vec{\lambda} ; \vec{\mu})_{k}\right\}_{k}$ of the space $B^{*}(\vec{\lambda} ; \vec{\mu})$. Then,

$$
\left\langle\left.\Psi_{\vec{\lambda}}^{G}(Z)\right|_{\alpha}=\sum_{\vec{\mu} \in b(\vec{\lambda})} \sum_{\beta, k} C(\vec{\lambda}, \vec{\mu})_{\alpha}^{\beta k} f(\vec{\lambda} ; \vec{\mu})_{k} \otimes\left\langle\left.\Psi_{\vec{\mu}}^{H}(Z)\right|_{\beta}\right.\right.
$$

where $C(\vec{\lambda}, \vec{\mu})_{\alpha}^{\beta k}$ are constants independent of coordinates $Z$.

Notice that sometimes the space of the conformal blocks of $\hat{h}$ WZW model with the states $\vec{\mu}$ is zero-dimensional and does not contributes to the summation of the r.h.s. in Eq. (2.8).

Let us explain the decomposition (2.8). Suppose that a vector $\left\langle\Upsilon_{\vec{\lambda}}^{G}(Z)\right| \in L_{\vec{\lambda}}^{\dagger}$ belongs to the $\hat{g}$-gauge invariant subspace $\mathscr{T}_{\vec{\lambda}}^{G \dagger}(\mathrm{Z})$, and has a decomposition $\varphi\left(\left\langle\Upsilon_{\vec{\lambda}}^{G}(Z)\right|\right)=\sum_{\vec{\mu}, k} f(\vec{\lambda} ; \vec{\mu})_{k} \otimes\left\langle\left.\Gamma_{\vec{\mu}}^{H}(Z)\right|^{k}\right.$ with $\left\langle\left.\Gamma_{\vec{\mu}}^{H}(Z)\right|^{k} \in L_{\vec{\mu}}^{\dagger}\right.$. For any current $J_{I}$ of $\hat{h} \subset \hat{g}$,

$$
\begin{aligned}
0 & =\left\langle\Upsilon_{\vec{\lambda}}^{G}(Z)\right| \sum_{i=1}^{N} \operatorname{Res}_{z_{\imath}}\left[\xi(w) \varrho_{i}\left(J_{I}(w)\right) d w\right] \\
& =\sum_{\vec{\mu}, k} f(\vec{\lambda} ; \vec{\mu})_{k} \otimes\left\langle\left.\Gamma_{\vec{\mu}}^{H}(Z)\right|^{k} \sum_{i=1}^{N} \operatorname{Res}_{z_{i}}\left[\xi(w) \varrho_{i}\left(J_{I}(w)\right) d w\right]\right.
\end{aligned}
$$




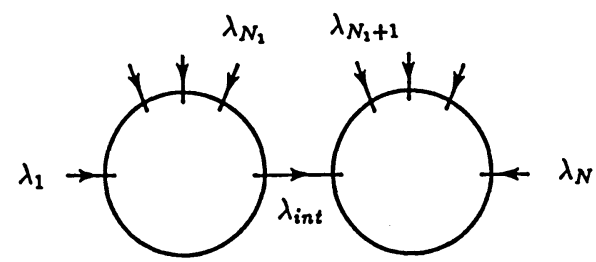

Fig. 1. A degenerate limit of the conformal blocks

From the linear independence of $f(\vec{\lambda} ; \vec{\mu})_{k}$ 's, it follows that $\left\langle\left.\Gamma_{\vec{\mu}}^{H}(Z)\right|^{k}\right.$ belongs to the $\hat{h}$-gauge invariant subspace $\mathscr{T}_{\vec{\mu}}^{H \dagger}(Z)$. Hence, the isomorphism (2.7) induces the inclusion

$$
\tilde{\varphi}: \mathscr{T}_{\vec{\lambda}}^{G \dagger}(Z) \hookrightarrow \bigoplus_{\vec{\mu} \in b(\vec{\lambda})} B^{*}(\vec{\lambda} ; \vec{\mu}) \otimes \mathscr{T}_{\vec{\mu}}^{H \dagger}(Z) .
$$

We thus obtain the decomposition of a base $\left\langle\left.\Psi_{\vec{\lambda}}^{G}(Z)\right|_{\alpha}\right.$ as in (2.8) except for the fact that the coefficients $C(\vec{\lambda}, \vec{\mu})_{\alpha}^{\beta k}$ might depend on the coordinate $Z$. Now recall the fact that the energy momentum tensors of $\hat{g}$ and $\hat{h}$ coincide. By considering the action of the operator $\operatorname{Res}_{z_{i}} \varrho_{i}(T(w) d w)$ and using (2.5), we obtain the equation

$$
0=\sum_{\vec{\mu} \in b(\vec{\lambda})} \sum_{\beta, k}\left(\partial_{z_{i}} C(\vec{\lambda}, \vec{\mu})_{\alpha}^{\beta k}\right) f(\vec{\lambda} ; \vec{\mu})_{k} \otimes\left\langle\left.\Psi_{\vec{\mu}}^{H}(Z)\right|_{\beta},\right.
$$

From the linear independence of the basis $f(\vec{\lambda}, \vec{\mu})_{k} \otimes\left\langle\left.\Psi_{\vec{\mu}}^{H}(Z)\right|_{\beta}\right.$, the coefficients $C(\vec{\lambda}, \vec{\mu})_{\alpha}^{\beta k}$ are indeed constants.

We remark here the commutativity of the factorization [FS, TUY] and the decomposition (2.8). Consider a degenerate limit where an $N$-points block splits into $N_{1}+1$ and $N_{2}+1$-points blocks $\left(N_{1}+N_{2}=N\right)$ as in Fig. 1. It induces a canonical isomorphism

$$
\text { Fac }: \mathscr{V}_{\vec{\lambda}}^{G \dagger} \cong \bigoplus_{\lambda_{\text {int }}} \mathscr{V}_{\left(\vec{\lambda}_{1}, \vec{\lambda}_{\text {int }}^{*}\right)}^{G \dagger} \otimes \mathscr{V}_{\left(\vec{\lambda}_{\text {int }}, \vec{\lambda}_{2}\right)}^{G \dagger},
$$

where

$$
\begin{aligned}
& \left(\vec{\lambda}_{1}, \lambda_{\text {int }}^{*}\right)=\left(\lambda_{1}, \ldots, \lambda_{N_{1}}, \lambda_{\text {int }}^{*}\right) \\
& \left(\lambda_{\text {int }}, \vec{\lambda}_{2}\right)=\left(\lambda_{\text {int }}, \lambda_{N_{1}+1}, \ldots, \lambda_{N}\right) .
\end{aligned}
$$

and $\lambda_{\text {int }}$ and $\lambda_{\text {int }}^{*}$ represent an intermediate state and its conjugate. Since the decomposition (2.8) is independent of $Z$, we have a following commutative diagram:

$$
\begin{aligned}
& \stackrel{\tilde{\varphi}}{\mathscr{T}_{\vec{\lambda}}^{G \dagger}} \stackrel{\tilde{\varphi}}{\longrightarrow} \underset{\vec{\mu} \in b(\vec{\lambda})}{\bigoplus_{\vec{\mu}} \mathscr{T}^{H \dagger}} \\
& \cong \downarrow \mathrm{Fac} \quad \cong \downarrow
\end{aligned}
$$

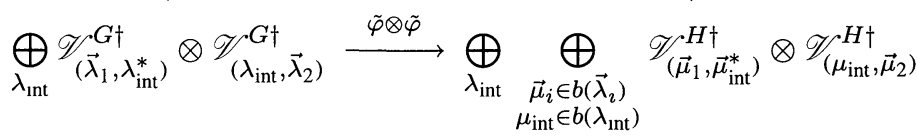

We shall extensively use this fact in the proof of our main theorem. 


\section{Branching Rule for $\widehat{g l}(l r)_{1} \supset \widehat{s l}(l)_{r} \times \widehat{s l}(r)_{l} \times \hat{a}$}

In this section we present the branching formula for the conformal embedding $\widehat{g l}(l r)_{1} \supset \widehat{s l}(l)_{r} \times \widehat{s l}(r)_{l} \times \hat{a}$ following [H]. This was also studied in [ABI, Wa].

\subsection{Fermionic Construction of $\widehat{g l}(l r)_{1}$ in the Neveu-Schwartz Sector}

The conformal embedding $\widehat{g l}(l r)_{1} \supset \widehat{s l}(l)_{r} \times \widehat{s l}(r)_{l} \times \hat{a}$ is most conveniently realized by considering the free fermions having $\operatorname{sl}(l) \times s l(r)$ symmetry. Consider the $l r$ fermions $\psi^{j p}(1 \leq j \leq l, 1 \leq p \leq r)$ and their conjugates $\psi_{j p}$. Here, the index $j(p)$ represents the vector representation of $s l(l)(s l(r))$, respectively. Throughout this paper, we assume the Neveu-Schwartz boundary condition

$$
\psi^{j p}(z)=\sum_{n \in Z+1 / 2} z^{-n-1 / 2} \psi^{j p}(n), \quad \psi_{j p}(z)=\sum_{n \in Z+1 / 2} z^{-n-1 / 2} \psi_{j p}(n) .
$$

The canonical commutation relations are given by

$$
\left\{\psi^{j p}(n), \psi_{k q}(m)\right\}=\delta_{k}^{j} \delta_{q}^{p} \delta_{m+n, 0} .
$$

The currents are expressed through the bilinear combinations of fermions as

$$
\begin{array}{lll}
\widehat{g l}(l r)_{1} & J_{k q}^{j p}(n)=\sum_{m}: \psi^{j p}(n-m) \psi_{k q}(m): \\
\widehat{s l}(l)_{r} \quad & J_{k}^{j}(n) \quad(j \neq k), \quad J_{j}^{j}(n)-J_{j+1}^{j+1}(n) \quad(j=1, \ldots, l-1), \\
& J_{k}^{j}(n)=\sum_{p=1}^{r} \sum_{m}: \psi^{j p}(n-m) \psi_{k p}(m):, \\
\widehat{s l}(r)_{l} \quad J_{q}^{p}(n) \quad(p \neq q), \quad J_{p}^{p}(n)-J_{p+1}^{p+1}(n) \quad(p=1, \ldots, r-1), & \\
& J_{q}^{p}(n)=\sum_{j=1}^{l} \sum_{m}: \psi^{j p}(n-m) \psi_{j q}(m): \\
\hat{a} & J(n)=\sum_{j=1}^{l} \sum_{p=1}^{r} \sum_{m}: \psi^{j p}(n-m) \psi_{j p}(m): .
\end{array}
$$

We are now interested in the representation of $\widehat{g l}(l r)_{1}$ on the fermion Fock space $F$ defined from the vacuum state $|0\rangle$ with

$$
\psi^{j p}(n)|0\rangle=\psi_{j p}(n)|0\rangle=0 \quad \text { for } \quad n>0 .
$$

With respect to the $\widehat{g l}(l r)_{1}$, the Fock space $F$ is reducible and decomposes as

$$
F=\sum_{p=-\infty}^{\infty} F_{p}
$$

where the $\hat{a}$-charge $p$ counts the number of fermion creation operators $\psi^{j p}(-n)$ minus the number of conjugate fermion creation operators $\psi_{j p}(-n)$ in a state over the 
vacuum. By the conformal embedding $\widehat{g l}(l r)_{1} \supset \widehat{s l}(l r)_{1} \times \hat{a}$, each space $F_{p}$ factorizes into

$$
F_{p} \cong L_{\Lambda_{p}} \otimes H_{p}
$$

where $\Lambda_{p}$ is the $p^{\text {th }}$ fundamental weight of $\widehat{s l}(l r)_{1}$ (with the convention $\Lambda_{p}=\Lambda_{p^{\prime}}$ if $\left.p \equiv p^{\prime} \bmod l r\right)$, and $H_{p}$ is the charge $p$ representation of the abelian part $\hat{a}$.

For $n \in \mathbf{Z}+\frac{1}{2}, 1 \leq j \leq l, 1 \leq p \leq r$, the state $s_{j p}(n)$ of energy $n$ is created by $\psi^{j p}(-n)$ and annihilated by $\psi_{j p}(n)$. The vacuum state $|0\rangle$ in Eq. (3.4) occupies all the negative energy states. There is an effective way to represent the basis elements of the Fock space using the 2-component Maya diagrams [H]. First, we introduce the infinite series of $l$ by $r$ rectangles $\left\{[l, r]_{n}\right\}$ labeled by $n \in \mathbf{Z}+\frac{1}{2}$. We arrange them in a row so that the right vertical edge of $[l, r]_{n}$ is attached to the left vertical edge of $[l, r r]_{n+1}$ as follows:

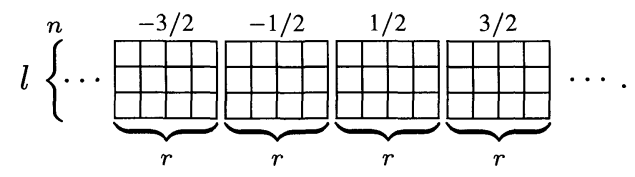

A 2-component Maya diagram $M$ is an ordered set

$$
\begin{array}{r}
M=\left\{m_{j p}(n) \mid m_{j p}(n)=\mathbb{Z} \text { or } \square\left(1 \leq j \leq l, 1 \leq p \leq r, n \in Z+\frac{1}{2}\right)\right. \\
\text { such that } \left.m_{j p}(n)=\mathbb{Q} \text { for } n \ll 0, m_{j p}(n)=\square \text { for } n \gg 0\right\},
\end{array}
$$

and $\mathscr{O}_{l}^{r}$ denotes the set of all the 2-component Maya diagrams. We shall identify $m_{j p}(n)$ of $M$ with the $(j, p)^{\text {th }}$ box of $[l, r]_{n}$. There is a one-to-one correspondence between the elements of $\mathscr{M}_{l}^{r}$ and the basis elements of the Fock space $F$ as

$$
M \mapsto \prod_{\substack{n<0 \\ m_{j p}(n)=\mathbb{Q}}} \psi^{j, p}(n) \prod_{\substack{n<0 \\ m_{j p}(n)=\square}} \psi_{j, p}(n)|0\rangle .
$$

(We do not mind the signature ambiguity from the ordering of the fermions.) In other words, we hatch the $(j, p)^{\text {th }}$ box in $[l, r]_{n}$ if the state $s_{j p}(n)$ is occupied, and we leave it blank, if the state $s_{j p}(n)$ is empty. Here, we give some examples in the case $l=2$, $r=3$.

$$
\begin{aligned}
\text { energy } & \\
|0\rangle & =\cdots \\
\psi^{1,3}\left(-\frac{3}{2}\right) \psi^{2,1}\left(-\frac{1}{2}\right)|0\rangle & =\cdots \\
\psi^{2,2}\left(-\frac{1}{2}\right) \psi_{2,3}\left(-\frac{3}{2}\right) \psi_{1,1}\left(-\frac{1}{2}\right)|0\rangle & =\cdots
\end{aligned}
$$

For a Maya diagram, the $\hat{a}$-current counts

(\# of hatched boxes above the Fermi sea level)

- (\# of blank boxes below the Fermi sea level).

The actions of the currents of $\widehat{s l}(l)_{r}$ and $\widehat{s l}(r)_{l}$ are also easily represented on Maya diagrams. The current $J_{k}^{j}(n)$ in (3.3), for example, annihilates the states in the $k^{\text {th }}$ row of $[l, r]_{m}$ and create the states in the $j^{\text {th }}$ row of $[l, r]_{m-n}$. We choose the triangular 
decomposition of $\widehat{s l}(l) \hat{s l}(r))$ as the currents $J_{k}^{j}(0)\left(J_{q}^{p}(0)\right)$ for $j>k(q>p)$ are the creation operators.

\subsection{Branching Rule of $F_{p}$}

The branching rule of $F_{p}$ for the conformal embedding $\widehat{g l}(l r)_{1} \supset \widehat{s l}(l)_{r} \times \widehat{s l}(r)_{l} \times \hat{a}$ is a special case of Theorem 4.2 in $[\mathrm{H}]$. (See also [ABI].) Let us rewrite the formula to the form which is convenient for our later use.

We introduce the set of "Young diagrams" $\mathscr{H}_{l}^{r}$ consists of sequences $Y=\left(y_{1}, \ldots, y_{l}\right)\left(y_{i} \in Z, y_{1} \geq \ldots \geq y_{l-1}\right)$ with the level $r$ constraint

$$
y_{1}-y_{l} \leq r \text {. }
$$

Let $|Y|=\sum_{j=1}^{l} \mathscr{Y}_{j}$ denote the "size" of the Young diagram $Y \in \mathscr{Y}_{l}^{r}$. There is an inclusion $\mathscr{H}_{l}^{r} \subset \mathscr{C}_{l}^{r}$ by the identification of a Young diagram $Y \in \mathscr{H}_{l}^{r}$ with a 2-component Maya diagram $M(Y) \in \mathscr{M}_{l}^{p}$ as

$$
m_{j p}(n)= \begin{cases}\square & \text { if } r\left(n-\frac{1}{2}\right)+p \leq y_{j} \\ \square & \text { otherwise }\end{cases}
$$

Through the correspondence (3.9), a Young diagram $Y$ is also identified with a basis element of the Fock space $F$ with $\hat{a}$-charge $|Y|$. Let us write this state $|Y\rangle$. For example, in the case $l=2, r=3$,

$$
\begin{array}{lll}
Y=(4,2) & (p=6) & \mapsto|Y\rangle=\cdots \\
Y=(-1,-4) & (p=-5) \mapsto|Y\rangle=\cdots
\end{array}
$$

The fundamental fact about the branching rule for the embedding (1.3) is the following one $[\mathrm{H}]:$ A state $|Y\rangle$ with $Y \in \mathscr{Y}_{l}^{r}$ is a highest weight state of subalgebra $\widehat{s l}(l)_{r} \times$ $\widehat{s l}(r)_{l} \times \hat{a}$, and conversely all the highest weight states of $\widehat{s l}(l)_{r} \times \widehat{s l}(r)_{l} \times \hat{a}$ are given by $|Y\rangle$ with $Y \in \mathscr{Y}_{l}^{r}$. Hence, we have a decomposition

$$
F_{p} \cong \bigoplus_{\substack{Y \in \mathscr{Y}_{l}^{r} \\|Y|=p}} F_{Y}
$$

where $F_{Y}$ is the irreducible $\widehat{s l}(l)_{r} \times \widehat{s l}(r)_{l} \times \hat{a}$-module of the highest weight state $|Y\rangle$.

To see what representation a highest weight state $|Y\rangle$ represents, we need some notations. Let $P_{+}(l, r)$ denote the set of dominant integral weights of $\widehat{s l}(l)_{r}$, and $\mathscr{Y} /-1, r$ denote the set of Young diagrams $Y=\left(y_{j}\right)_{j=1}^{l-1}\left(r \geq y_{1} \geq \ldots \geq y_{l-1} \geq 0\right)$ contained in the rectangle $[l-1, r]$ with height $l-1$ and width $r$. Then, there is a bijection $\lambda: \mathscr{H}_{l-1, r} \rightarrow P_{+}(l, r)$ as

$$
\lambda(Y)=\left(r-y_{1}+y_{l}\right) \Lambda_{0}+\sum_{j=1}^{l-1}\left(y_{j}-y_{j+1}\right) \Lambda_{j},
$$

where we insert the dummy variable $y_{l}=0$. Under this correspondence, the Young diagram $Y$ represents the classical part of the dominant integral weights $\lambda(Y)$. We extend the bijection $\lambda$ to the map $\lambda: \mathscr{Y}_{l}^{r} \rightarrow P_{+}(l, r)$ through Eq. (3.16). 
The transposition of Maya diagrams $t: \mathscr{M}_{l}^{r} \rightarrow \mathscr{M}_{r}^{l}$ is defined by setting $m_{p j}(n)=$ $m_{j p}(n)$, that is, by the transposition along NW-SE diagonal of each reactangle $[l, r]_{n}$. The transposition of Young diagrams $t: \mathscr{H}_{l}^{r} \rightarrow \mathscr{Y}_{r}^{l}$ is then obtained under the embedding $\mathscr{F}_{l}^{r} \subset \mathscr{M}_{l}^{r}$ of (3.13), where that the level $r$ constraint (3.12) insures that the obtained diagram is a Young diagram with a level $l$ constraint. We simply write the image $t(Y)$ as ${ }^{t} Y$. For example, in the case $l=3, r=4$, the transposition of the Young diagram $Y=(10,8,7) \in \mathscr{Y}_{3}^{4}$ is ${ }^{t} Y=(7,7,6,5)$ as

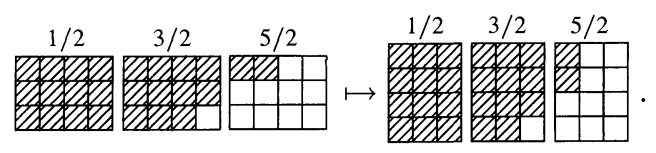

We thus obtain a diagram

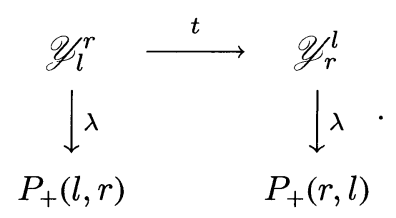

We use the same symbol $Y$ for its image $\lambda(Y)$ if there is no confusion. In this notation, the second fundamental fact is that $[\mathrm{H}]$ for any $Y \in \mathscr{Y}_{l}^{r}$, the state $|Y\rangle$ is the highest state of the representation $Y \otimes{ }^{t} Y \otimes(|Y|)$ of $\widehat{s l}(l)_{r} \otimes \widehat{s l}(r)_{l} \otimes \hat{a}$. Thus, the branching formula of $F_{p}$ becomes as follows:

$$
F_{p} \cong\left[\sum_{\substack{Y \in \mathscr{P}_{l}^{r} \\|Y|=p}} L_{Y} \otimes L_{t}\right] \otimes H_{p}
$$

where $L_{Y}$ represents the irreducible module of $Y$.

We introduce a subset $\mathscr{Y}_{0 l}^{r} \subset \mathscr{Y}_{l}^{r}$ with the condition $0 \leq y_{l} \leq r-1$ for $\left(y_{j}\right) \in \mathscr{Y}_{0 l}^{r}$. We write the Young diagram $(r, \ldots, r)$ as $\left(r^{l}\right)$. Let us define the addition of the two Young diagrams $Y=\left(y_{j}\right), Y^{\prime}=\left(y_{j}^{\prime}\right)$ as $Y+Y^{\prime}=\left(y_{j}+y_{j}^{\prime}\right)$. For any Young diagram $Y \in \mathscr{Y}_{l}^{r}$, there is a unique pair $\left(Y^{0}, K\right)$, where $Y^{0} \in \mathscr{Y}_{0 l}^{r}, K \in \mathbf{Z}$ such that $Y=Y^{0}+K\left(r^{l}\right)$. Thus, there is a decomposition

$$
\mathscr{H}_{l}^{r} \cong \mathscr{H}_{0 l}^{r} \times \mathbf{Z}
$$

From Eq. (3.16), $\lambda(Y)=\lambda\left(Y^{0}\right), \lambda\left({ }^{t} Y\right)=\lambda\left({ }^{t}\left(Y^{0}\right)\right)$. It follows that the $\widehat{s l}(l)_{r} \times \widehat{s l}(r)_{l}$ part depends only on $p$ modulo $l r$, and that the decomposition has another expression as

$$
F_{p} \cong\left[\sum_{Y \in b(p)} L_{Y} \otimes L_{t}\right] \otimes H_{p}
$$

where

$$
b(p)=\left\{Y\left|Y \in \mathscr{Y}_{0 l}^{r},\right| Y \mid \equiv p \bmod l r\right\} .
$$


Let us illustrate the above branching rule (3.21) by the example $l=2, r=3$.

$$
\begin{aligned}
& p \equiv 0:(\emptyset \otimes \emptyset+\square \square \otimes \square) \otimes(p), \\
& p \equiv 1:(\square \otimes \square+\square \square \square \otimes \square) \otimes(p), \\
& p \equiv 2:(\square \otimes \square+\square \otimes \boxminus) \otimes(p), \\
& p \equiv 3:(\square \otimes \square+\square \square \otimes \boxminus) \otimes(p), \\
& p \equiv 4:(\boxminus \otimes \boxminus+\square \otimes \square) \otimes(p), \\
& p \equiv 5:(\square \square \square \otimes \boxplus+\square(p) \text {. }
\end{aligned}
$$

\section{Complete Pairing Between Conformal Blocks}

We shall apply the general formalism in Sect. 2 to the particular case of our interest $\widehat{g l}(l r)_{1} \supset \widehat{s l}(l)_{r} \times \widehat{s l}(r)_{l} \times \hat{a}$. A significant fact is that the dimension of the space of the conformal blocks for $\widehat{g l}(l r)_{1}$ and $\hat{a}$ on the Riemann sphere are at most one, i.e.

$$
\operatorname{dim} \mathscr{V}_{\vec{p}}^{G \dagger}=\operatorname{dim} \mathscr{V}_{\vec{p}}^{A \dagger}= \begin{cases}1 & \text { if } p_{1}+\ldots+p_{N}=0, \\ 0 & \text { otherwise }\end{cases}
$$

Let us choose the basis of the space of conformal blocks as follows:

$$
\begin{array}{cl}
\widehat{g l}(l r)_{1} & :\left\langle\Phi_{\vec{p}}^{G}\right|, \\
\widehat{s l}(l)_{r} & :\left\langle\left.\Phi_{\vec{\lambda}}^{L}\right|_{\alpha},\right. \\
\widehat{s l}(r)_{l} & :\left\langle\left.\Phi_{\vec{\lambda}}^{R}\right|_{\alpha},\right. \\
\hat{a} & :\left\langle\Phi_{\vec{p}}^{A}\right| .
\end{array}
$$

Here the index $\alpha$ distinguishes the multiplicity if the dimension of $\mathscr{V}_{\vec{\lambda}}^{L \dagger}$ or $\mathscr{V}_{\vec{\lambda}}^{R \dagger}$ is greater than one. Using these bases, the base $\left\langle\Phi_{\vec{p}}^{G}\right|$ is expanded as

$$
\left\langle\Phi_{\vec{p}}^{G}\right|=\sum_{\vec{Y} \in b(\vec{p})} \sum_{\alpha, \beta} C(\vec{p}, \vec{Y})_{\alpha \beta}\left\langle\Phi _ { \vec { Y } } ^ { L } | _ { \alpha } \otimes \left\langle\left.\Phi_{t \vec{Y}}^{R}\right|_{\beta} \otimes\left\langle\Phi_{\vec{p}}^{A}\right|,\right.\right.
$$

where $b(\vec{p})=\left\{\vec{Y}=Y_{1}, \ldots, Y_{N}\right) \mid Y_{i} \in b\left(p_{i}\right)$ for every $\left.i\right\}$.

The coefficient matrix $C(\vec{p}, \vec{Y})_{\alpha \beta}$ defines a pairing between the spaces of conformal blocks $\mathscr{V}_{\vec{Y}}^{L}$ and $\mathscr{T}_{t \vec{Y}}^{R}$. The following theorem states that this pairing is in fact complete. Thus it determines the dual basis of $\mathscr{V}_{\vec{Y}}^{L \dagger}$ from a basis of $\mathscr{V}_{t \vec{Y}}^{R \dagger}$ and vice versa.

Theorem. Fix $\vec{p}$ with $\sum_{i=1}^{N} p_{i}=0$ and $\vec{Y} \in b(\vec{p})$. Then,

1) The dimensions of $\mathscr{V}_{\vec{Y}}^{L \dagger}$ and $\mathscr{V}_{\vec{t}}^{R \dagger}$ are equal.

2) For the coefficient matrix $C(\vec{p}, \vec{Y})_{\alpha \beta}$ in Eq. (4.3),

$$
\operatorname{det} C(\vec{p}, \vec{Y})_{\alpha \beta} \neq 0,
$$

where the determinant is taken with respect to $\alpha, \beta$. 
The proof of the theorem will be given in the next section.

Remark. We can show that for $\vec{p}, \vec{q}$ with $\Sigma p_{i}=\Sigma q_{i}=0$ and $\vec{p}-\vec{q} \in(\operatorname{lr} \mathbf{Z})^{N}$, there is a constant $c_{\vec{p} \vec{q}}$ independent of $\alpha, \beta$ such that $C(\vec{p}, \vec{Y})_{\alpha \beta}=c_{\vec{p} \vec{q}} C(\vec{q}, \vec{Y})_{\alpha \beta}$. Thus, $C(\vec{p}, \vec{Y})_{\alpha \beta}$ provides a canonical pairing between $\mathscr{V}_{\vec{Y}}^{L \dagger}$ and $\mathscr{T}_{t \vec{Y}}^{R \dagger}$ independent of the choice of $\vec{p} \bmod (\operatorname{lr} \mathbf{Z})^{N}$.

For $Y=\left(y_{j}\right) \in \mathscr{Y}_{l}^{r}$ the conjugate of $\lambda(Y) \otimes \lambda\left({ }^{t} Y\right)$ is given by $\lambda\left(Y^{*}\right) \otimes \lambda\left({ }^{t}\left(Y^{*}\right)\right)$, where $Y^{*} \in \mathscr{Y}_{l}^{r}$ is defined as

$$
Y^{*}=\left(-y_{l}, \ldots,-y_{1}\right)
$$

For $Y \in \mathscr{H}_{0 l}^{r}$ its conjugate $Y^{*} \in \mathscr{H}_{0 l}^{r}$ are defined through Eq. (3.20), or explicitly,

$$
Y^{*}=\left\{\begin{array}{ll}
\left(r-y_{l}, \ldots, r-y_{1}\right) & \text { if } 0 \leq y_{1} \leq r \\
\left(2 r-y_{l}, \ldots, 2 r-y_{1}\right) & \text { if } r<y_{1} \leq 2 r-1
\end{array} .\right.
$$

For the special case of 3-point functions, Theorem 1 reduces to the fusion rule duality in Eq. (1.2).

Corollary. For any triplet $\vec{Y}=\left(Y_{1}, Y_{2}, Y_{3}^{*}\right)$ such that $Y_{i} \in \mathscr{Y}_{0 l}^{r}$ and $\left|Y_{1}\right|+\left|Y_{2}\right| \equiv$ $\left|Y_{3}\right| \bmod l r$, and its transposition ${ }^{t} \vec{Y}=\left({ }^{t} Y_{1},{ }^{t} Y_{2},{ }^{t} Y_{3}^{*}\right)$, the equality

$$
\operatorname{dim} \mathscr{V}_{\vec{Y}}^{L \dagger}=\operatorname{dim} \mathscr{V}_{t \vec{Y}}^{R \dagger}
$$

holds.

Proof. Because of the fact $\left|Y_{3}\right|+\left|Y_{3}^{*}\right| \equiv 0 \bmod l r$ and the condition $\left|Y_{1}\right|+\left|Y_{2}^{*}\right| \equiv$ $\left|Y_{3}\right| \bmod l r$, there is a triplet $\vec{p}=\left(p_{1}, p_{2}, p_{3}\right)$ satisfies i) $\vec{Y} \in b(\vec{p})$, ii) $p_{1}+p_{2}+p_{3}=0$. Then the equality (4.7) immediately follows from the theorem. q.e.d.

Let us see some examples of the dual pairing of conformal blocks in our familiar example $l=2, r=3$. For a triplet $\vec{\lambda}=(\square, \square, \emptyset)$ of $\widehat{s l}(2)_{3}, \operatorname{dim} \mathscr{V}_{\vec{\lambda}}^{{ }^{\dagger \dagger}}=1$. (The Young diagrams here represent the classical part of $\lambda_{i}$ 's.) For each representation $\lambda_{i}$, there are three choices of $p_{i} \bmod 6$ such that $Y_{i} \in b\left(p_{i}\right)$ and $\lambda\left(Y_{i}\right)=\lambda_{i}$. Accordingly, there are nine triplets $\vec{\lambda}^{\prime}$ of $\widehat{s l}(3)_{2}$ whose space of conformal blocks $\mathscr{V}_{\vec{\lambda}^{\prime}}^{R \dagger}$ are dual to $\mathscr{V}_{\vec{\lambda}}^{L \dagger}$. We present them below

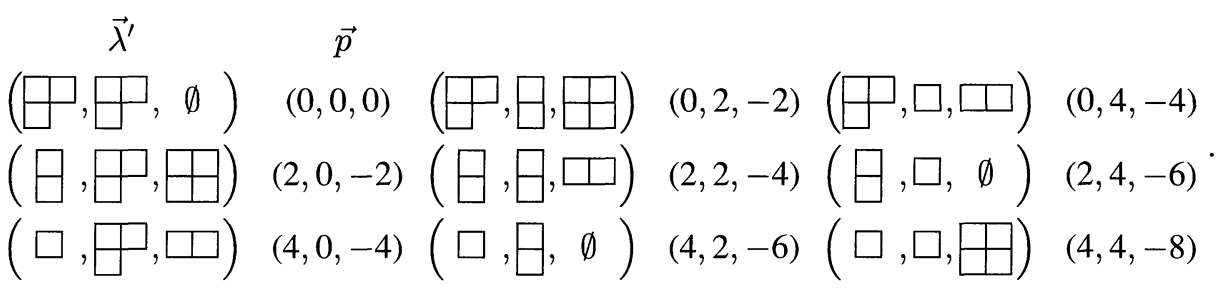

There is a canonical isomorphism between any two of them through the pairing with $\mathscr{V}_{\vec{\lambda}}^{L \dagger}$.

In general, there is a $\mathbf{Z}_{l}$-Dynkin automorphism of $\widehat{s l}(l)_{r}$ generated by

$$
\sigma: \Lambda_{i} \mapsto \Lambda_{i+1}
$$


For a Young diagram $Y=\left(y_{j}\right) \in \mathscr{H}_{l}^{r}$, we define $\sigma(Y)=\left(y_{j}^{\prime}\right)$, where

$$
y_{1}^{\prime}=y_{l}+r, \quad y_{j}^{\prime}=y_{j-1}(2 \leq j \leq l) .
$$

Then, $\lambda(\sigma(Y))=\sigma(\lambda(Y))$. For $Y \in \mathscr{Y}_{0 l}^{r}, \sigma(Y) \in \mathscr{Y}_{0 l}^{r}$ are defined through Eq. (3.20), or explicitly,

$$
\begin{array}{llll}
y_{1}^{\prime}=y_{l}+r, & y_{j}^{\prime}=y_{j-1}(2 \leq j \leq l) & \text { if } & 0 \leq y_{l-1} \leq r-1 \\
y_{1}^{\prime}=y_{l}, & y_{j}^{\prime}=y_{j-1}-r(2 \leq j \leq l) & \text { if } \quad r \leq y_{l-1} \leq 2 r-1
\end{array}
$$

Noticing the fact that for $Y \in \mathscr{Y}_{0 l}^{r}, \lambda\left({ }^{t}(\sigma(Y))\right)=\lambda\left({ }^{t} Y\right)$ and $|\sigma(Y)| \equiv|Y|+r \bmod l r$,

Corollary. Let $\sigma_{1}, \ldots, \sigma_{N}$ be elements of the $\mathbf{Z}_{l}$-automorphism of $\widehat{s l}(l)_{r}$ with

$$
\prod_{i}^{N} \sigma_{i}=\mathrm{id}
$$

Fix $\vec{p}$ such that $\Sigma p_{i}=0$. Then, for the sets of Young diagrams in $\mathscr{H}_{0 l}^{r}$,

$$
\vec{Y}=\left(Y_{1}, \ldots, Y_{N}\right) \in b(\vec{p}), \quad \vec{X}=\left(\sigma_{1}\left(Y_{1}\right), \ldots, \sigma_{N}\left(Y_{N}\right)\right)
$$

there is a canonical isomorphism between the spaces of conformal blocks $\mathscr{T}_{\vec{Y}}^{L \dagger}$ and $\mathscr{T}_{\vec{X}}^{L \dagger}$ through the pairing with their common dual spaces $\mathscr{T}_{t_{\vec{Y}}}^{R \dagger}$.

\subsection{Braid Duality}

Another application of our theorem is giving relations between the braid matrices of $\widehat{s l}(l)_{r}$ and $\hat{s l}(r)_{l}$ WZW models as already pointed out by [Fu, NS, NRS, SA].

The braid group $B_{N}$ acts on the total space of conformal blocks of $\hat{g}$ :

$$
\mathscr{V}^{G \dagger}=\bigoplus_{\vec{\lambda}} \mathscr{V}_{\vec{\lambda}}^{G \dagger}
$$

A generator $B_{i j}$ interchanges the points $z_{i}$ and $z_{j}$ and the representations $\lambda_{i}$ and $\lambda_{j}$ by the analytic continuation counterlockwise. We choose bases for $\widehat{g l}(l)_{1}$ and $\hat{a}$ as in the appendix. As for $\widehat{s l}(l)_{r}$ and $\widehat{s l}(r)_{l}$, we choose dual bases w.r.t. the form $C(\vec{p}, \vec{Y})$, that is Eq. (4.3) becomes a diagonal form

$$
\left\langle\Phi_{\vec{p}}^{G}\right|=\sum_{\vec{Y} \in b(\vec{p})} \sum_{\alpha}\left\langle\Phi _ { \vec { Y } } ^ { L } | _ { \alpha } \otimes \left\langle\left.\Phi_{t \vec{Y}}^{R}\right|_{\alpha} \otimes\left\langle\Phi_{\vec{p}}^{A}\right| .\right.\right.
$$

The braid generator $B_{i j}$ acts on these basis elements as follows:

$$
\begin{aligned}
&\left\langle\Phi_{\vec{p}}^{G}\right| \mapsto K\left(\vec{p}, \sigma_{i j}(\vec{p})\right)\left\langle\Phi_{\sigma_{\imath j}(\vec{p})}^{G}\right|, \\
&\left\langle\Phi _ { \vec { Y } } ^ { L } | _ { \alpha } \mapsto \Sigma _ { \beta } M ( \vec { Y } , \sigma _ { i j } ( \vec { Y } ) ) _ { \alpha \beta } \left\langle\left.\Phi_{\sigma_{\imath j}(\vec{Y})}^{L}\right|_{\beta},\right.\right. \\
&\left\langle\Phi _ { t \vec { Y } } ^ { R } | _ { \alpha } \mapsto \Sigma _ { \beta } N ( { } ^ { t } \vec { Y } , \sigma _ { i j } ( { } ^ { t } \vec { Y } ) ) _ { \alpha \beta } \left\langle\left.\Phi_{\left.\sigma_{i j}(t \vec{Y})\right)}^{R}\right|_{\beta},\right.\right. \\
&\left\langle\Phi_{\vec{p}}^{A}\right| \mapsto L\left(\vec{p}, \sigma_{i j}(\vec{p})\right)\left\langle\Phi_{\sigma_{i j}(\vec{p})}^{A}\right|,
\end{aligned}
$$

where $\sigma_{i j}(\vec{\lambda})$ is defined from $\vec{\lambda}$ by exchanging its $i^{\text {th }}$ and $j^{\text {th }}$ representations. Then, 
Proposition. For any $\vec{Y} \in b(\vec{p})$, the braid matrices in (4.14) satisfy the following relation

$$
\sum_{\alpha} M\left(\vec{Y}, \sigma_{i j}(\vec{Y})\right)_{\alpha \beta} N\left({ }^{t} \vec{Y}, \sigma_{i j}\left({ }^{t} \vec{Y}\right)\right)_{\alpha \gamma}=\delta_{\beta \gamma} K\left(\vec{p}, \sigma_{i j}(\vec{p})\right) L\left(\vec{p}, \sigma_{i j}(\vec{p})\right)^{-1} .
$$

If $p_{i}=p_{j}$, the space of conformal blocks $\mathscr{T}_{\vec{p}}^{G \dagger}$ and $\mathscr{V}_{\vec{p}}^{A \dagger}$ are stable under the action of $B_{i j}$. Their braid matrices are calculated from the explicit form of the conformal blocks in the appendix, or by using the knowledge of the conformal dimensions of the highest states, as

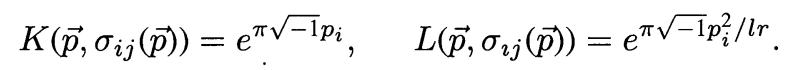

In particular, in the case $\vec{Y}=\left(Y_{1}, \ldots, Y_{N}\right)$ such that $Y_{1}=\ldots=Y_{N-1} \in b(p)$, the above proposition states that dual spaces $\mathscr{V}_{\vec{Y}}^{L \dagger}$ and $\mathscr{V}_{t \vec{Y}}^{R \dagger}$ obey the braid group $B_{N-1}$ representations contragradient to each other up to a phase factor $e^{\pi \sqrt{-1}\left(p-p^{2} / l r\right)}$.

\section{Proof of the Theorem}

Before giving a proof, we prepare the known facts about the dimensionality of the spaces of conformal blocks, or the fusion rule, of $\widehat{s l}(l)_{r}$.

For $\vec{\lambda}=\left(\lambda_{1},(r-1) \Lambda_{0}+\Lambda_{1}, \lambda_{2}^{*}\right)$ such that $\lambda_{1}, \lambda_{2} \in P_{+}(l, r)$, the fusion rules are known to be

$$
\operatorname{dim} \mathscr{V}_{\vec{\lambda}}^{L \dagger}= \begin{cases}1 & \text { if } \lambda_{2}-\lambda_{1}=\Lambda_{i+1}-\Lambda_{i} \text { for some } i \\ 0 & \text { otherwise. }\end{cases}
$$

For $Y \in \mathscr{Y}_{0 l}^{r}$ and an integer $N=|Y|+K l r$ with $K \geq 0$ being an integer, a restricted path of length $N$ from $\square$ to $Y$ is a series of Young diagrams $Y_{1}=\square$, $Y_{2}, \ldots, Y_{N}=Y+K\left(r^{l}\right) \in \mathscr{Y}_{l}^{r}$ with $\left|Y_{i+1}\right|=\left|Y_{i}\right|+1$ such that $Y_{i+1}$ is obtained by adding a box to $Y_{i}$. Using Eq. (5.1) and the factorization property of the conformal blocks in a degenerate limit of Fig. 2 ,

Proposition 1. For $\vec{Y}=(\overbrace{\square, \ldots, \square}^{N}, Y^{*})$ with $Y \in \mathscr{Y}_{0 l}^{r}$ and $N=|Y|+K$ lr with an integer $K \geq 0$

$$
\operatorname{dim} \mathscr{T}_{\vec{Y}}^{L \dagger}=(\# \text { of the restricted paths of length } N \text { from } \square \text { to } Y)
$$

Moreover, the braid group $B_{N}$ acts irreducibly on $\mathscr{V}_{\vec{Y}}^{L \dagger}[\mathrm{TK}, \mathrm{KT}, \mathrm{We}]$.

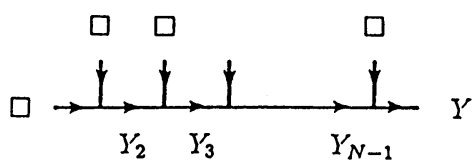

Fig. 2. In this degenerate limit, there is a one-to-one correspondence between the basis elements of the conformal blocks and the restricted paths 
Because there is a bijection between the set of the restricted paths of lenth $N$ and $\square$ to $Y$ in $\mathscr{F}_{l}^{r}$ to the set of the restricted paths of length $N$ from $\square$ to ${ }^{t} Y$ in $\mathscr{Y}_{r}^{l}$ by the transposition (3.18), it follows from Proposition 1 that [JMO]

Proposition 2. For $\vec{Y}=(\overbrace{\square, \ldots, \square}^{N}, Y^{*})$ with $Y \in \mathscr{Y}_{0 l}^{r}$ and $N=|Y|+K$ lr with an integer $K \geq 0$,

$$
\operatorname{dim} \mathscr{T}_{\vec{Y}}^{L \dagger}=\operatorname{dim} \mathscr{V}_{t \vec{Y}}^{R \dagger} .
$$

5.1. The Case $\vec{p}=\left(1, \ldots, 1,-K_{1} l r,-K_{2} l r,-K_{3} l r\right)$

Let $K_{1}, K_{2}, K_{3}$ be non-negative integers. Let

$$
\vec{p}_{f}=(\overbrace{1, \ldots, 1}^{N},-K_{1} l r,-K_{2} l r,-K_{3} l r)
$$

with $N=\left(K_{1}+K_{2}+K_{3}\right) l r$. From the branching rule (3.16), the expansion of $\left\langle\Phi_{\vec{p}_{f}}^{G}\right|$ includes the terms

$$
\sum_{\alpha, \beta} C\left(\vec{p}_{f}, \vec{Y}_{f}\right)_{\alpha \beta}\left\langle\Phi _ { \vec { Y } _ { f } } ^ { L } | _ { \alpha } \otimes \left\langle\left.\Phi_{t_{Y_{f}}}^{R}\right|_{\beta} \otimes\left\langle\Phi_{\vec{p}_{f}}^{A}\right|\right.\right.
$$

for

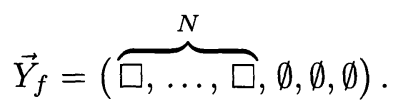

In this subsection, we concentrate on this special type of conformal blocks.

Lemma 3. Let $\vec{p}_{f}$ and $\vec{Y}_{f}$ be as above. Then, the coefficient matrix $C\left(\vec{p}_{f}, \vec{Y}_{f}\right)_{\alpha \beta}$ is non-zero matrix.

Proof. Using the prescription in Sect.3.2, the highest states have the following Fock space representations:

$$
\begin{gathered}
\square \otimes \square \otimes(1)=\psi^{1,1}\left(-\frac{1}{2}\right)|0\rangle, \\
\emptyset \otimes \emptyset \otimes\left(-K_{i} l r\right)=\prod_{m, j, p} \psi_{j, p}\left(-\frac{1}{2}-m\right)|0\rangle,
\end{gathered}
$$

where the product is over $1 \leq m \leq K_{i}, 1 \leq j \leq l, 1 \leq p \leq r$, and we do not mind the signature ambiguity from the ordering of the fermion operators. Notice that $\square \otimes \square \otimes(1)$ has the elements

$$
\psi^{j, p}\left(-\frac{1}{2}\right)|0\rangle, \quad 1 \leq j \leq l, 1 \leq p \leq r
$$

as its descendant states as a $\widehat{s l}(l)_{r} \times \widehat{s l}(r)_{l} \times \hat{a}$ module.

We rename the preceding $N$ points $z s_{1}, \ldots, z_{N}$ as $z_{m j p}\left(1 \leq m \leq K_{1}+K_{2}+K_{3}\right.$, $1 \leq j \leq l, 1 \leq p \leq r)$, and evaluate the following quantity for $\mathscr{T}_{\vec{p}_{f}}^{G \dagger}$ :

$$
\begin{aligned}
\left\langle\Phi_{\vec{p}_{f}}^{G}\right. & \mid\left[\bigotimes_{m, j, p}\left[\psi^{j, p}\left(-\frac{1}{2}\right)|0\rangle_{m j p}\right] \otimes\left[\prod_{m, j, p} \psi_{j, p}\left(-\frac{1}{2}-m\right)|0\rangle_{N+1}\right]\right. \\
\otimes & {\left.\left[\prod_{m, j, p} \psi_{j, p}\left(-\frac{1}{2}-m\right)|0\rangle_{N+2}\right] \otimes\left[\prod_{m, j, p} \psi_{j, p}\left(-\frac{1}{2}-m\right)|0\rangle_{N+3}\right]\right], }
\end{aligned}
$$


where the subscription of the vacuum refers to the insertion points $z_{m j p}, z_{N+i}$. This is evaluated by using the explicit form of $\left\langle\Phi_{\vec{p}_{f}}^{G}\right|$ in the appendix, and indeed a nonvanishing function. q.e.d.

Lemma 4. Let $\vec{p}_{f}$ and $\vec{Y}_{f}$ are as same as in Lemma 3. Then,
1) $\operatorname{dim} \mathscr{V}_{\vec{Y}_{f}}^{\dagger}=\operatorname{dim} \mathscr{V}_{t_{\vec{Y}_{f}}}^{\dagger}$
2) $\operatorname{det} C\left(\vec{p}_{f}, \vec{Y}_{f}\right) \neq 0$.

Proof. The first part can be reduced to a special case of Proposition 2. To show the second part, we consider the action of the braid group $B_{N}$, which interchanges the positions of points $z_{i}(i=1, \ldots, N)$, on both sides of (4.3). Because of (4.1), the actions of the braid group on $\mathscr{V}_{\vec{p}_{f}}^{G \dagger}$ and $\mathscr{V}_{\vec{p}_{f}}^{A \dagger}$ are trivial up to the phase factor. (This phase factor is $e^{2 \pi \sqrt{-1}(1-1 / l r)}$ by (4.18), though we do not need to know its exact value here.) On the other hand, the space of $\mathscr{T}_{\vec{Y}_{f}}^{L \dagger} \otimes \mathscr{T}_{\vec{Y}_{f}}^{R \dagger}$ closes under $B_{N}$. Thus, by Lemma 3 the space $\mathscr{V}_{\vec{Y}_{f}}^{L \dagger} \otimes \mathscr{T}_{t \vec{Y}_{f}}^{R \dagger}$ is equipped with the non-vanishing bilinear form $C\left(\vec{p}_{f}, \vec{Y}_{f}\right)_{\alpha \beta}$ invariant by the braid group $B_{N}$ up to a phase factor $e^{2 \pi \sqrt{-1}(1-1 / l f)}$. From the irreducibility of the braid representations on $\mathscr{T}_{\vec{Y}_{f}}^{L \dagger}$ in Proposition 1 and by using Schur's lemma, it follows that the braid representations on $\mathscr{V}_{\vec{Y}_{f}}^{L \dagger}$ and $\mathscr{T}_{{ }_{\vec{Y}_{f}}}^{R \dagger}$ are contragradient to each other and that the coefficient matrix should be complete, i.e., $\operatorname{det} C\left(\vec{p}_{j}, \vec{Y}_{j}\right) \neq 0$. q.e.d.

\subsection{Proof of the General Case}

We show the theorem in the case of three-point functions. We take a degerate limit of the basis element $\left\langle\Phi_{\vec{p}_{f}}^{G}\right|$ for $\vec{p}_{f}$ of (5.4) as in Fig. 3a. Three intermediate states appearing at the vertex are denoted by $\vec{p}=\left(p_{1}, p_{2}, p_{3}\right)$. Correspondingly, consider the degenerate limit of $\left\langle\left.\Phi_{\vec{Y}_{f}}^{L}\right|_{\alpha} \otimes\left\langle\left.\Phi_{t_{Y_{f}}}^{R}\right|_{\beta} \otimes\left\langle\Phi_{\vec{p}_{f}}^{A}\right|\right.\right.$ for $\vec{Y}_{f}$ of (5.6) as Fig. 3b. (We only write the $\widehat{s l}(l)_{r}$ part in the figure.) Then the set of three intermediate states $\vec{Y}=\left(Y_{1}, Y_{2}, Y_{3}\right)$ are an element of $b(\vec{p})$. Conversely,

$\mathbf{a}$

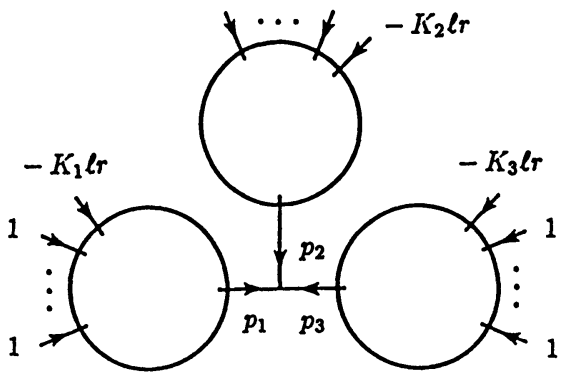

b

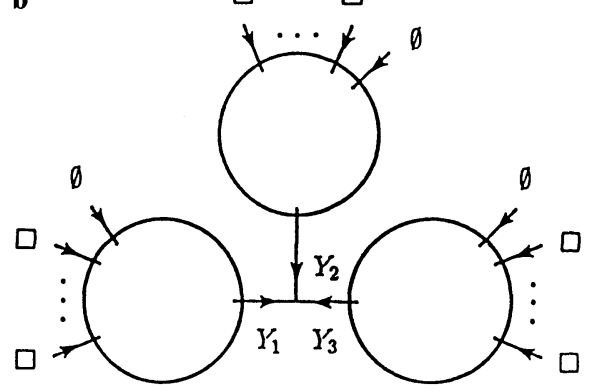

Fig. 3a, b. A degenerate limit of $\mathscr{\mathscr { p }}_{f}^{G \dagger}$ and $\mathscr{V}_{\vec{Y}_{f}}^{G \dagger}$ 
Lemma 5. For given $\vec{p}=\left(p_{1}, p_{2}, p_{3}\right)$ with $p_{1}+p_{2}+p_{3}=0$ and $\vec{Y} \in b(\vec{p})$, there exist some non-negative integers $K_{1}, K_{2}, K_{3}$ such that $\vec{p}$ and $\vec{Y}$ are realized as the intermediate states of $\mathscr{V}_{\vec{p}}$ and $\mathscr{T}_{\vec{Y}}$ respectively under the degenerate limit as in Fig. 3.

Proof. If $\left|Y_{i}\right|<p_{i}$, we set $K_{i}=0$ and obtain $Y_{i}$ by the fusion of $p_{i} \square$ 's. If $\left|Y_{i}\right| \geq p_{i}$, we set $K_{i}=\left(|Y|-p_{i}\right) / l r$ and obtain $Y_{i}$ by the fusion of $\left|Y_{i}\right| \square$ 's q.e.d.

Proof of the theorem. Now, we prove the theorem combining Proposition 2, Lemmas 4 and 5. For given $\vec{p}_{0}=\left(p_{1}, p_{2}, p_{3}\right)$ with $p_{1}+p_{2}+p_{3}=0$ and $\vec{Y}_{0}=\left(Y_{1}, Y_{2}, Y_{3}\right) \in b\left(\vec{p}_{0}\right)$, set $\vec{p}_{f}$ and $\vec{Y}_{f}$ as (5.4), (5.6) and choose integers $K_{1}, K_{2}, K_{3}$ as in Lemma 5. By the construction, the factorization under the degenerate limit of Fig. 3 gives

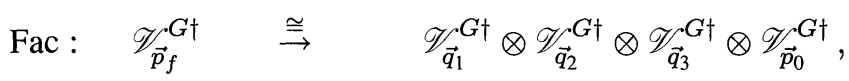

$$
\begin{aligned}
& \text { Fac : } \mathscr{T}_{\vec{Y}_{f}}^{L \dagger} \otimes \mathscr{V}_{t_{\vec{Y}_{f}}}^{R \dagger} \cong \underset{\vec{Y} \in b\left(\vec{p}_{0}\right)}{\bigoplus}\left(\mathscr{T}_{\vec{X}_{1}}^{L \dagger} \otimes \mathscr{T}_{t_{\vec{X}_{1}}}^{R \dagger}\right) \otimes\left(\mathscr{V}_{\vec{X}_{2}}^{L \dagger} \otimes \mathscr{T}_{t_{\vec{X}_{2}}}^{R \dagger}\right) \\
& \otimes\left(\mathscr{V}_{\vec{X}_{3}}^{L \dagger} \otimes \mathscr{T}_{t_{\vec{X}_{3}}^{R \dagger}}^{R+}\right) \otimes\left(\mathscr{V}_{\vec{Y}}^{L \dagger} \otimes \mathscr{V}_{t \vec{Y}}^{R \dagger}\right)
\end{aligned}
$$

where

$$
\begin{aligned}
\vec{q}_{i} & =(\overbrace{1, \ldots, 1}^{p_{i}+K_{2} l r},-K_{i} l r,-p_{i}), \\
\vec{X}_{i} & =(\overbrace{\square, \ldots, \square}^{p_{i}+K_{i} l r}, \emptyset, Y_{i}^{*}) .
\end{aligned}
$$

For a fixed intermediate state $\vec{Y}$, let $B^{L}(\vec{Y})$ denote a basis of $\mathscr{V}_{\vec{X}_{1}}^{L \dagger} \otimes \mathscr{V}_{\vec{X}_{2}}^{L \dagger} \otimes \mathscr{V}_{\vec{X}_{3}}^{L \dagger} \otimes \mathscr{T}_{\vec{Y}}^{L \dagger}$ obtained by the tensor product of bases of its components. Also let $B^{R}\left({ }^{t} \vec{Y}\right)$ denote the set of tensor products of bases of $\mathscr{T}_{t_{\vec{X}_{1}}^{R \dagger}}^{R{ }^{2}} \mathscr{T}_{t_{\vec{X}_{2}}}^{R \dagger} \otimes \mathscr{T}_{{ }_{\vec{X}_{3}}^{R \dagger}}^{R} \otimes \mathscr{V}_{t_{\vec{Y}}}^{R \dagger}$. The set

$$
\mathbf{B}=\left\{\Phi_{\alpha} \otimes \Psi_{\beta} \mid \Phi_{\alpha} \in B^{L}(\vec{Y}), \Psi_{\beta} \in B^{R}\left({ }^{t} \vec{Y}\right), \vec{Y} \in b(\vec{p})\right\}
$$

gives a basis of $\mathscr{V}_{\vec{Y}_{f}}^{L \dagger} \otimes \mathscr{V}_{t_{\vec{Y}_{f}}}^{R \dagger}$ through the isomorphism (5.10). In this choice of a basis, the elements of the coefficient matrix are

$$
C\left(\vec{p}_{f}, \vec{Y}_{f}\right)_{\alpha \beta}=0
$$

for basis element $\Phi_{\alpha} \in B^{L}(\vec{Y})$ and $\Psi_{\beta} \in B^{R}(\vec{X})$ if $\vec{X} \neq{ }^{t} \vec{Y}$. In other words, the coefficient matrix $C\left(\vec{p}_{f}, \vec{Y}_{f}\right)_{\alpha \beta}$ has a following block structure:

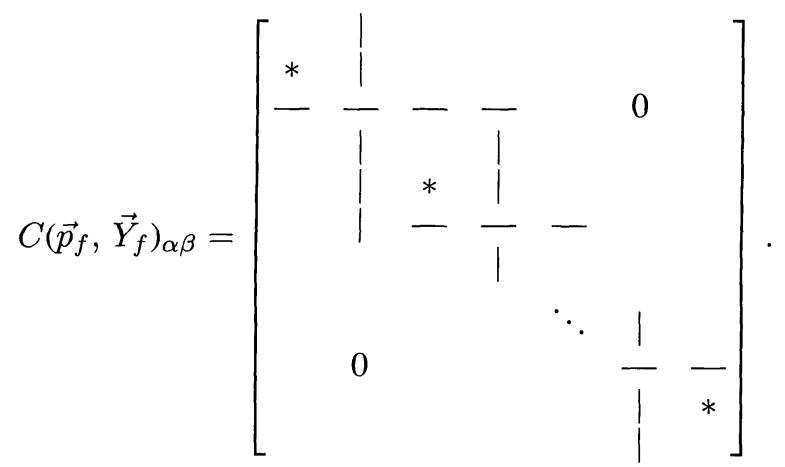


Since $\operatorname{det} C\left(\vec{p}_{f}, \vec{Y}_{f}\right) \neq 0$ by Lemma 4 , it follows that

$$
\operatorname{dim} \mathscr{V}_{\vec{X}_{1}}^{L \dagger} \otimes \mathscr{V}_{\vec{X}_{2}}^{L \dagger} \otimes \mathscr{T}_{\vec{X}_{3}}^{L \dagger} \otimes \mathscr{V}_{\vec{Y}}^{L \dagger}=\operatorname{dim} \mathscr{V}_{t_{\vec{X}_{1}}}^{R \dagger} \otimes \mathscr{T}_{t_{\mathbb{X}_{2}}}^{R \dagger} \otimes \mathscr{V}_{t_{\vec{X}_{3}}}^{R \dagger} \otimes \mathscr{V}_{t_{\vec{Y}}}^{R \dagger}
$$

From Proposition 2,

$$
\operatorname{dim} \mathscr{V}_{\vec{X}_{i}}^{L \dagger}=\operatorname{dim} \mathscr{V}_{t_{\vec{X}_{\imath}}}^{R \dagger} \quad(i=1,2,3)
$$

so we obtain

$$
\operatorname{dim} \mathscr{V}_{\vec{Y}}^{L \dagger}=\operatorname{dim} \mathscr{V}_{t \vec{Y}}^{R \dagger}
$$

Furthermore, the restriction of the matrix $C\left(\vec{p}_{f}, \vec{Y}_{f}\right)$ to the square sub-matrix acting on the subspace spanned by $B^{L}(\vec{Y}) \otimes B^{R}\left({ }^{t} \vec{Y}\right)$ has a following factorized form:

$$
C(\vec{p}, \vec{Y})_{\alpha_{0} \beta_{0}} C\left(\vec{q}_{1}, \vec{Y}_{1}\right)_{\alpha_{1} \beta_{1}} C\left(\vec{q}_{2}, \vec{Y}_{2}\right)_{\alpha_{2} \beta_{2}} C\left(\vec{q}_{3}, \vec{Y}_{3}\right)_{\alpha_{3} \beta_{3}}
$$

because of our choice of the basis and the commutativity in Eq. (2.14). Thus, we have arrived at the formula

$$
\begin{aligned}
\operatorname{det} C\left(\vec{p}_{f}, \vec{Y}_{f}\right)_{\alpha \beta}= & \prod_{\vec{Y} \in b(\vec{p})} \operatorname{det} C(\vec{p}, \vec{Y})_{\alpha_{0} \beta_{0}} \operatorname{det} C\left(\vec{q}_{1}, \vec{Y}_{1}\right)_{\alpha_{1} \beta_{1}} \\
& \times \operatorname{det} C\left(\vec{q}_{2}, \vec{Y}_{2}\right)_{\alpha_{2} \beta_{2}} \operatorname{det} C\left(\vec{q}_{3}, \vec{Y}_{3}\right)_{\alpha_{3} \beta_{3}} .
\end{aligned}
$$

In the product the determinant is understood to be 1 if the corresponding space of the conformal blocks is zero dimensional. Because the 1.h.s. of Eq. (5.19) is nonzero from Lemma 4 , it follows that $\operatorname{det} C\left(\vec{p}_{0}, \vec{Y}_{0}\right)_{\alpha_{0} \beta_{0}} \neq 0$. Thus, we have shown the theorem for three-point functions.

For general $N$-point functions, we can obtain a factorization formula for the determinant of the coefficient matrices by those of the 3-point functions similar to Eq. (5.19) in a particular degenerate limit. Thus, their determinant is non-vanishing. This completes the proof of the theorem.

\section{Discussion}

We have shown that there is a complete pairing, or the level-rank duality, between the conformal blocks of $\widehat{s l}(l)_{r}$ and $\widehat{s l}(r)_{l}$ WZW models on the Riemann sphere induced from the decompositions under the conformal embedding (1.3). The duality, as its nature, provides us only a relation of both models and cannot determine the exact behavior of each model directly. However, if we have some knowledge about one theory, we can extract a hidden or unexpected structure of the other theory through the duality. For example, the $l$-reduction property in the $\widehat{s l}(l)_{r}$ WZW model turns into a Dynkin automorphism property in the $\widehat{s l}(r)_{l}$ WZW model as we saw in the corollary of Sect. 4.1. We left such applications of the duality as a future problem, and we shall discuss further extensions of our formulation of the duality in the rest of the paper.

The reciprocal decomposition formulas are also known for other types of algebras $(C, C),(B, B),(B, D),(D, D)[\mathrm{H}]$ (for $(C, C)$ case [KP]). It seems possible to repeat the analysis along the lines presented in this paper. The easiest case is the pair

$$
\widehat{s p}(2 l)_{r} \times \widehat{s p}(2 r)_{l} \subset \hat{o}(4 l r)_{1}
$$


bcause there is no Dynkin automorphism for $\widehat{s p}$ and no spin representations. Again, we consider the Neveu-Schwartz fermions

$$
\psi^{j p}(z)=\sum_{n \in \mathbf{Z}+1 / 2} z^{-n-1 / 2} \psi^{j p}(n), \quad(j= \pm 1, \ldots, \pm l, p= \pm 1, \ldots, \pm r)
$$

with the canonical commutation relations

$$
\left\{\psi^{j p}(m), \psi^{k q}(n)\right\}=\delta_{j+k, 0} \delta_{p+q, 0} \delta_{m+n, 0} .
$$

This time, the fermion Fock space $F$ decomposes into two $\hat{o}$-irreducible modules as $F_{+} \otimes F_{-}$where $F_{+}\left(F_{-}\right)$consists of the fermion states with even (odd) fermion number. The reciprocal decomposition formula for (6.1) is actually much simpler than $(A, A)$ case, and given by $[\mathrm{H}]$

$$
F_{+} \cong \sum_{\substack{Y \in \mathscr{Y} l, r \\|Y| \text { :even }}} L_{Y} \otimes L_{t_{Y}}, \quad F_{-} \cong \sum_{\substack{Y \in \mathscr{Y} /, r \\|Y| \text { :odd }}} L_{Y} \otimes L_{t_{Y}}
$$

where $\mathscr{H}_{l, r}$ is the set of all the Young diagrams in the rectangle $[l, r]$. From this formula, we deduce that the dual pairing occurs again between the spaces $\mathscr{V}_{\vec{Y}}^{L \dagger}$ of $\widehat{s p}(2 l)_{r}$ and $\mathscr{V}_{t \vec{Y}}^{R \dagger}$ of $\widehat{s p}(2 r)_{l}$. In our proof of the completeness of the pairing in the $A$-type case, the following two properties are essential:

1) The braid representation of the conformal blocks is irreducible if the external states are those of the vector representation.

2) Any integrable representation is produced by the fusions of the vector representations.

For $C$-type WZW models, these facts still hold [KT], so that the level-rank duality exists. We remark that if we choose the Ramond boundary condition for the fermions instead of Eq. (6.2), not the pair $\left(Y,{ }^{t} Y\right)$ but the pair $\left(Y,\left({ }^{t} Y\right)^{*}\right)$ appears in the decomposition formula $[\mathrm{H}]$.

Unfortunately, both properties 1) and 2) fail for o-type pairs $(B, B),(B, D),(D, D)$ because of the spinor representations. However, one can modify the situation if we add a Dynkin automorphism to the generator of the algebra, and restrict the external states to those of the representations produced by vector representations. This condition is equivalent to the one that the classical part of the representations have the lifts for $O(n)$ representations. Then the completeness of the pairing could again be preserved. It needs further investigation to justify these statements and also to include the spin representations as well.

Though we have restricted our attention to the conformal blocks on the Riemann sphere so far, it is possible to extend the duality to the conformal blocks on the higher genus Riemann surfaces through the factorization to the three point blocks. Though the principle of our construction remains valid, there are two major differences between genus zero and other cases. One is the appearance of an infinite number of intermediate states along the cycles for $\widehat{g l}$ and $\hat{a}$ WZW models, or, the infinite dimension on the conformal blocks. It is a characteristic property of the abelian theories. The other is that the complete pairing occurs not between the spaces of conformal blocks themselves but between their subspaces.

To describe the situation, let us consider the zero-point function on the torus. It is useful to add a set of "charge shifting" currents (see the appendix for the field $X(z)$ )

$$
V_{n}(z)=: e^{n l r X(z)}:, \quad n \in \mathbf{Z}
$$


both to $\hat{a}$ and to $\widehat{g l}(l r)_{1}$ and make the dimensions of conformal blocks finite. We write this new algebra $\hat{a}^{\prime}$ and $\widehat{g l}^{\prime}(l r)_{1}$. Then,

$$
\widehat{s l}(l)_{r} \times \widehat{s l}(r)_{l} \times \hat{a}^{\prime} \subset \widehat{g l}^{\prime}(l r)_{1},
$$

is still a conformal embedding. The dimension of the conformal blocks of zeropoint functions of $\widehat{g l}^{\prime}(l r)_{1}$ are $l r$. From the rule (3.16), each block or the character decomposes as

$$
c h^{G^{\prime}}(p)=\sum_{Y \in b(p)} \operatorname{ch}^{L}(Y) \operatorname{ch}^{R}\left({ }^{t} Y\right) \operatorname{ch}^{A^{\prime}}(\|Y\|), \quad(0 \leq p \leq l r-1)
$$

where $c h^{G^{\prime}}, c h^{L}, c h^{R}, c h^{A^{\prime}}$ represent the characters of the $\widehat{g l}^{\prime}(l r)_{1}, \widehat{s l}(l)_{r}, \widehat{s l}(r)_{l}, \hat{a}^{\prime}$ WZW models, and $\|Y\|$ denotes the integer $0 \leq j \leq l r-1$ with $j \equiv|Y| \bmod l r$. For example, for $l=2, r=3$, it induces the following pairing between the spaces of the conformal blocks of zero-point functions of $\widehat{s l}(2)_{3}$ and $\widehat{s l}(3)_{2}$

$$
C(\overrightarrow{0}, \overrightarrow{0})_{\alpha \beta}^{g=1}=\left(\begin{array}{cc|c|ccc|cc}
1 & \square & \square & \square & \square & G \\
& 1 & & 1 & & 1 \\
\hline 1 & & 1 & & 1 & \\
& 1 & & 1 & & 1
\end{array}\right) \begin{gathered}
\emptyset \\
\square
\end{gathered} .
$$

As is obvious from this example, the complete pairings appear in the several combinations between the subspaces of the both spaces of conformal blocks for the general Riemann surface with genus greater than zero. This complication is originated to the Dynkin automorphism property of the $A$-type, and it does not appear for the $C$-type case.

For the duality, it is natural to add the fermions to $\widehat{g l}(l r)_{1}$ as additional currents [KNTY, Wi2] because the dimensions of the conformal blocks again become at most one for a fixed spin structure. Remarkably, the modular transformations which do not change the spin structure act trivially on the space of the conformal blocks up to a phase factor. As a corollary, we can deduce a relation between the $S$-matrices of the $\widehat{s l}(l)_{r}$ and $\widehat{s l}(r)_{l}$ conformal blocks, which is an analogue of the braid matrix relation (4.17). We note that such a relation has already appeared in Corollary $6.2 \mathrm{~b}$ in $[\mathrm{H}]$.

Acknowledgements. Many details of the duality were realized through discussions with A. Kuniba. We would like to give sincere thanks to him. We also thank K. Hasegawa and Y. Yamada for useful discussions.

\section{Appendix. Conformal Blocks of $\widehat{g l}(l r)_{1}$ and $\hat{a}$ WZW Models}

The conformal blocks of $\widehat{g l}(l r)_{1}$ and $\hat{a}$ WZW models are equivalent to the correlation functions of free fermions and bosons respectively in the following way. Consult [FMS, P] for the issues on fermion and boson correlators and the state-field correspondence. 
The case $\widehat{g l}(l r)_{1}$. We write the fermion operators $\psi_{k q}(n), \psi^{k q}(n)$ acting on the $i^{\text {th }}$ Fock spaces of

$$
\sum_{\vec{p}} F_{p_{1}} \otimes \ldots \otimes F_{p_{N}}
$$

as $\psi_{k q, i}(n), \psi^{k q}{ }_{, i}(n)$. The fermion operators are understood to be anticommuting when $i \neq j$. The identification of the basis elements is defined as

$$
\begin{aligned}
|s\rangle & =f_{1}|0\rangle_{1} \otimes \ldots \otimes f_{N}|0\rangle_{N} \\
& =f_{1} \ldots f_{N}|0\rangle
\end{aligned}
$$

with $f_{i}$ being a product of the operators $\psi_{k q, i}(-n), \psi^{k q}{ }_{, i}(-n)\left(i\right.$ : fixed). Let $B_{m n}^{i j}(Z)$ be the coefficients of the expansion

$$
\left(z-z_{i}\right)^{-m-\frac{1}{2}} \sqrt{d z}=\sum_{n>0} B_{m n}^{i j}(Z)\left(z-z_{j}\right)^{n-\frac{1}{2}} \sqrt{d z}
$$

or, explicitly,

$$
B_{m n}^{\imath \jmath}(Z)=\frac{1}{\left(m-\frac{1}{2}\right) !} \frac{1}{\left(n-\frac{1}{2}\right) !} \partial_{z_{\jmath}}^{n-\frac{1}{2}} \partial_{z_{i}}^{m-\frac{1}{2}}\left(z_{j}-z_{i}\right)^{-1}
$$

Using them, a basis element of conformal blocks is given by

$$
\langle\Phi(Z)|=\sum_{\substack{\vec{p} \in Z^{N} \\ \Sigma p_{i}=0}}\left\langle\Phi_{\vec{p}}(Z)\right|=\langle 0| G(Z),
$$

where $\langle 0|$ is the vacuum state in $F_{0}^{\dagger} \otimes \ldots \otimes F_{0}^{\dagger}$ and

$$
G(Z)=\exp \left[\sum_{k, q} \sum_{\substack{1 \leq i, j \leq N \\ i \neq j}} \sum_{m, n>0} B_{m n}^{i j}(Z) \psi^{k q}, i(m) \psi_{k q, j}(n)\right] .
$$

The conditions (2.4-5) are proved by the following relation $(n>0)$ :

$$
\begin{aligned}
& G(Z) \psi_{k q, i}(-n) G(Z)^{-1}=\psi_{k q, i}(-n)+\sum_{\substack{j=1 \\
j \neq i}}^{N} \sum_{m>0} B_{m n}^{j i} \psi_{k q, j}(m), \\
& G(Z) \psi_{, i}^{k q}(-n) G(Z)^{-1}=\psi_{, i}^{k q}(-n)+\sum_{\substack{j=1 \\
j \neq i}}^{N} \sum_{m>0} B_{m n}^{j i} \psi_{, j}^{k q}(m) .
\end{aligned}
$$

Here we used the property $B_{m n}^{i j}=-B_{n m}^{j i}$. Then the conformal block is evaluated as

$$
\langle 0|G(Z)| s\rangle
$$

with the help of (A.7). This is equivalent to the usual Wick expansion rule of the free fermion field under the correspondence

$\psi_{k q, i}(-n) \leftrightarrow \frac{1}{\left(n-\frac{1}{2}\right) !} \partial^{n-\frac{1}{2}} \psi_{k q}\left(z_{i}\right), \quad \psi^{k q, i}(-n) \leftrightarrow \frac{1}{\left(n-\frac{1}{2}\right) !} \partial^{n-\frac{1}{2}} \psi^{k q}\left(z_{i}\right)$ 
Namely, if the state $\left|s_{i}\right\rangle$ corresponds to the field $\Psi_{i}$, then, the conformal block evaluated by the states

$$
|s\rangle=\left|s_{1}\right\rangle \otimes \ldots \otimes\left|s_{N}\right\rangle
$$

equals to

$$
\left\langle\Psi_{1}\left(z_{1}\right) \ldots \Psi_{N}\left(z_{N}\right)\right\rangle_{F},
$$

where $\langle\cdots\rangle_{F}$ means the free fermion correlation function.

The case $\hat{a}$. Again we introduce coefficients $C_{m n}^{i j}$ as

$$
\left(z-z_{i}\right)^{-m-1} d z=\sum_{n \geq 1} n l r C_{m n}^{i j}(Z)\left(z-z_{j}\right)^{n-1} d z
$$

or

$$
C_{m n}^{i j}(Z)=\frac{1}{l r} \frac{1}{m !} \frac{1}{n !} \partial_{z_{\jmath}}^{n} \partial_{z_{i}}^{m} \log \left(z_{j}-z_{i}\right)
$$

We extend the definition of $C_{m n}^{i j}(Z)$ through (A.13) if $m$ and/or $n$ are zero. Let us introduce scalar operators $X_{, i}$ conjugate to $J_{, i}(0)$ by

$$
\left[J_{, i}(0), X_{, j}\right]=\delta_{i, j} .
$$

A basis element of conformal blocks is given by

$$
\left\langle\Phi_{\vec{p}}(Z)\right|=\langle\vec{p}| G^{\prime}(Z),
$$

where $\langle\vec{p}|=\langle 0| e^{-\Sigma_{i} p_{i} X_{, i}}$ is the vacuum state in $H_{p_{1}}^{\dagger} \otimes \ldots \otimes H_{p_{N}}^{\dagger}$ and

$$
G^{\prime}(Z)=\exp \left[\frac{1}{2} \sum_{\substack{1 \leq i, j \leq N \\ i \neq j}} \sum_{m, n \geq 0} C_{m n}^{i j}(Z) J_{, i}(m) J_{, j}(n)\right] .
$$

The conformal block is evaluated by using the following relations:

$$
\begin{aligned}
G^{\prime}(Z) J_{, i}(-n) G^{\prime}(Z)^{-1} & =J_{, i}(-n)+\sum_{\substack{j=1 \\
j \neq i}}^{N} \sum_{m \geq 0} C_{m n}^{j i} J_{, j}(m), \quad(n>0), \\
G^{\prime}(Z) e^{p_{i} X_{, i}} G^{\prime}(Z)^{-1} & =e^{p_{\imath} X_{, i}} \times \exp \left[p_{i} \sum_{\substack{j=1 \\
j \neq i}}^{N} \sum_{m \geq 0} n l r C_{m 0}^{j i} J_{, j}(m)\right] .
\end{aligned}
$$

In evaluating the conformal block using (A.17), we choose a branch of the function

$$
\prod_{1 \leq j<i \leq N}\left(z_{j}-z_{i}\right)^{J_{, i}(0) J_{, j}(0) / l r}
$$

so that they are real values if $z_{i}$ 's are on the real axis as $z_{1}>\ldots>z_{N}>0$.

Again there is a state-field correspondence

$$
J_{, i}(-n) \leftrightarrow \frac{l r}{n !} \partial^{n} X\left(z_{i}\right), \quad e^{p_{\imath} X_{, \imath}} \leftrightarrow: e^{p_{\imath} X\left(z_{\imath}\right)}:,
$$

where $X(z)$ is a free scalar field with a propagator

$$
\langle X(z) X(w)\rangle=\frac{1}{l r} \log (z-w),
$$

and $J(z)=\operatorname{lr} \partial X(z)$. 


\section{References}

[ABI] Altschuler, D., Bauer, M., Itzykson, C.: Commun. Math. Phys. 132, 349 (1990)

[ABS] Altschuler, D., Bauer, M., Saleur, H.: J. Phys. A. Math. Gen. 32, L789 (1990)

[BB] Bais, F.A., Bouwknegt, P.: Nucl. Phys. B 279, 561 (1987)

[BETZ] Bais, F.A., Englert, F., Taormina, A., Zizzi, P.: Nucl. Phys. B 279, 529 (1987)

[BR] Bazhanov, V.V., Reshetikhin, N.: J. Phys. A. Math. Gen. 23, 1477 (1990)

[F] Frenkel, I.B.: In: Lect. Note in Math. vol.933, p. 71. Berlin, Heidelberg, New York: Springer 1982

[Fu] Fuchs, J.: Z. Phys. C 35, 89 (1987)

[FD] Fuchs, J., van Driel, P.: J. Math. Phys. 31, 1770 (1990)

[FMS] Friedan, D., Martinec, E., Shenkar, S.: Nucl. Phys. B 271, 93 (1986)

[FS] Friedan, D., Shenker, S.: Nucl. Phys. B 281, 509 (1987)

[G] Gepner, D.: Nucl. Phys. B 252, 481 (1985)

[GO] Goddard, P., Olive, D.: Nucl. Phys. B 257 [FS14], 226 (1985)

[GNO] Goddard, P., Nahm, W., Olive, D.: Phys. Lett. B 160, 111 (1985)

[GR] Gonzales, D., Redlich, A.N.: Phys. Lett. B 147, 150 (1984); Nucl. Phys. B 256, 621 (1985)

[GWe] Goodman, F.M., Wenzl, H.: Adv. Math. 82, 244 (1990)

[GWi] Gepner, D., Witten, E.: Nucl. Phys. B 278, 493 (1986)

[H] Hasegawa, K.: Publ. RIMS 25, 741 (1989)

[JM] Jimbo, M., Miwa, T.: Adv. Stud. Pure Math. 6, 17 (1985)

[JMO] Jimbo, M., Miwa, T., Okado, M.: Lett. Math. Phys. 14, 123 (1987); Nucl. Phys. 300, [FS22], 74 (1988)

[KN] Kuiba, A., Nakanishi, T.: In: Proc. of International Colloquium on Modern Quantum Field Theory. Bombay, Jan. 1990. Singapore: World Scientific 1991

[KNS] Kuniba, A., Nakanishi, T., Suzuki, J.: Nucl. Phys. B 356, 750 (1991)

[KNTY] Kawamoto, N., Namikawa, Y., Tsuchiya, A., Yamada, Y.: Commun. Math. Phys. 116, 247 (1988)

[KP] Kac, V.G., Peterson, D.: Proc. Natl. Acad. Sci. USA 78, 3308 (1981)

[KT] Kanie, Y., Tsuchiya, A.: In preparation

[KZ] Knizhnik, V., Zamolodchikov, A.B.: Nucl. Phys. B 247, 83 (1984)

[MS] Moore, G., Seiberg, N.: Commun. Math. Phys. 123, 177 (1989)

[NS] Naculich, S.G., Schnitzer, H.J.: Phys. Lett. B 244, 235 (1990)

[NRS] Naculich, S.G., Riggs, H.A., Schnitzer, H.J.: Phys. Lett. B 246, 417 (1990)

[P] Polyakov, A.M.: Gauge fields and strings. Chur: Harwood Academic 1987

[SA] Saleur, H., Altschler,D.: Nucl. Phys. B 354, 579 (1991)

[SW] Schellekens, A.N., Warner, N.P.: Phys. Rev. D 34, 3092 (1987)

[TK] Tsuchiya, A., Kanie, Y.: Adv. Stud. Pure Math. 16, 297 (1988)

[TUY] Tsuchiya, A., Ueno, K., Yamada, Y.: Adv. Stud. Pure Math. 19, 459 (1989)

[Wa] Walton, M.A.: Nucl. Phys. B 322, 775 (1989)

[We] Wenzl, H.: Inv. Math. 92, 349 (1988)

[Wi] Witten, E.: Commun. Math. Phys. 92, 455 (1984)

[Wi2] Witten, E.: Commun. Math. Phys. 113, 529 (1988) 